\title{
Barbara Galant \\ Uniwersytet Łódzki \\ LOS MARCADORES \\ DISCURSIVOS PUES \\ Y BUENO DESDE EL PRISMA \\ DE SU TRADUCCIÓN \\ AL POLACO
}

\section{Discourse markers pues and bueno and their translation into Polish}

\begin{abstract}
This article analyses the translations of two Spanish discourse markers - pues and bueno - into Polish based on excerpts from five novels by contemporary writers (Juan Marsé, Eduardo Mendoza, Carmen Posadas) and from a graphic novel by Alfonso Zapico. The theoretical part defines these linking words, outlines their common characteristics, as well as describes and illustrates with examples the functions of pues and bueno which play an important part in the cohesion and progression of a monologue or a dialogue. In the analytical section the pragmatic roles of both markers, used together in the originals, are analysed in order to see if they were rendered correctly in the translated versions.
\end{abstract}

KEYWORDS: discourse markers, pragmatic functions, translation, pues, bueno.

INTRODUCCIÓN

Este artículo se centrará en los marcadores discursivos españoles, tan recurrentes en la modalidad coloquial y conversacional, de entre los cuales se han escogido dos de muy alta frecuencia, pues y bueno que, además, pueden aparecer juntos en un enunciado. En la parte teórica se describirán los usos de dichas partículas para poder comprobar en la parte práctica si las mismas conservan su valor pragmático ${ }^{1}$ en la traducción al polaco, pues las soluciones prefabricadas que ofrecen los diccionarios bilingües no siempre se muestran válidas.

Antes de pasar al análisis de las funciones de dichas partículas cabe señalar que, aunque los marcadores discursivos se escapan a una categorización estricta por su natu-

1 Ya que abogamos por la teoría de Wittgenstein ([1953] 1999; citado por Otaola Olano 2004: 180) según la cual "el significado de una palabra es su uso en el lenguaje", consideramos que el significado sistemático del vocablo está inextricablemente vinculado a su uso. Es más, el famoso consejo del filósofo austríaco "no preguntéis por el significado, preguntad por el uso" (Wittgenstein [1953] 1999; citado por Otaola Olano 2004: 180) nos parece aún más pertinente a la hora de estudiar los marcadores discursivos. 
raleza heterogénea ${ }^{2}$, comparten las siguientes características tal y como afirma Portolés (1998: 25-26):

Los marcadores del discurso son unidades lingüísticas invariables, no ejercen una función sintáctica en el marco de la predicación oracional y poseen un cometido coincidente en el discurso: el de guiar, de acuerdo con sus distintas propiedades morfosintácticas, semánticas y pragmáticas, las inferencias que se realizan en la comunicación.

Si bien existen varias clasificaciones de marcadores ${ }^{3}$, Briz (Briz y Hildago 2008: 124) propone una sencilla división en conectores argumentativos que han de enlazar un monólogo o un diálogo y en conectores metadiscursivos que juegan un papel significativo en la cohesión y progresión de una conversación. Tanto pues como bueno pertenecen a la segunda clase, aunque pues, en su función de conector consecutivo, cabe en el primer grupo.

Cabe añadir que además de las aportaciones de los dos estudiosos mencionados anteriormente, existen numerosos estudios dedicados al significado, sintaxis y uso de las partículas discursivas, entre las cuales conviene destacar Diccionario de partículas de Santos Río (2003) o los trabajos de Martín Zorraquino (2008) y Montolío Durán (2008).

USO DE PUES

En cuanto a las funciones pragmáticas de pues, se pueden diferenciar tres valores: conector consecutivo, comentador y marcador metadiscursivo.

En este primer uso, lógicamente, dicho marcador sirve para indicar tanto la consecuencia como la continuidad y está situado, bien en el medio de una oración (entre comas), bien al inicio de esta que constituye una continuación de la anterior, lo cual se puede observar el siguiente ejemplo (Calvi, Mapelli 2004):

(1) Repito, pues, que no me interesa.

(2) ¿No quieres oír mis consejos? Pues llorarás algún día.

Además de marcar la consecuencia, pues, que se utiliza tanto en la oración principal como en la subordinada, puede hacer hincapié en la relación condicional, como se puede observar en los siguientes ejemplos (Calvi, Mapelli 2004):

(3) Si no quieres ir al cine, pues no vayas.

Con la función de comentador, el marcador anteriormente citado empieza una oración o incluso una intervención e introduce un comentario relevante al tema de la conversación (ej. 4), una reacción a una orden o pregunta (ej. 5) (López Quero 2007: 98). De acuerdo con López Quero (2007: 99), en los casos de una respuesta opuesta a las expectativas del interlocutor (ej. 6 de Martín Zorraquino y Portolés 1999: 4083-4084), pues bos.

2 Es un grupo compuesto por, entre otros, conjunciones, preposiciones, adverbios, interjecciones, ver-

3 Véase: Portolés y Martín Zorraquino 1999: 4080-4082 o Martín Zorraquino 2008: 52-53. 
"apoya el acto cortés" y permite, al mismo tiempo, expresar una opinión "más o menos cooperativa", pero en todo caso cooperativa.

(4) H1: ¿Usted conocía a Soleiro?

H2: ¿Un cojo $[\ldots]$ ?

H1: ¡El mismo! Pues se le apareció a la mujer en figura de cuervo.

(5) H1: [...] k más sueles leer?

$\mathrm{H} 2$ : pues en cuestion de comics, mortadelo y filemon ${ }^{4}$

(6) H1: [...] Ahora quiero que hablemos de este asunto.

H2: ¡Pues yo no!

H1: ¡Pues yo sí!

Pues, en calidad de metadiscursivo, desempeña un papel análogo al comentado en el párrafo anterior, visto que enfatiza el acuerdo o el desacuerdo, de ahí que suelan acompañarle adverbios como claro, sí, no (ejemplo 7 proporcionado por López Quero 2007: 96). Cuando este aparece solo, constituye una respuesta categórica (ejemplo 8 tomado de Calvi y Mapelli 2004). En cambio, en una interrogación, es el sinónimo de ¿por qué? (ej. 9) o un intento de animar el interlocutor a responder o a terminar su discurso (Calvi, Mapelli 2004). Del mismo modo, usado junto con un imperativo, normalmente después de este, invita a actuar (ej. 10 de las mismas autoras).

(7) ¿H1: Has visto todas sus películas?

$\mathrm{H} 2$ : pues no

(8) H1: ¿Conque habló mal de él?

H2: Pues.

(9) H1: No iré a su casa.

H2: ¿Pues?

(10) Vamos pues.

Por último, tal y como señalan Briz e Hidalgo (2008: 126-128, 133), pues como metadiscursivo constituye una pausa (hasta convertirse en una partícula de puntuación como en el ej. 11 que proporciona tiempo para dar con la forma idónea de expresar los pensamientos, pero también recomienza un diálogo después de un silencio, permite volver al tema interrumpido (ej. 12) o sirve para tomar la palabra (ej. 13):

(11) y me dice pues conoces a un tío y te mola pues por qué no te vas a enrollar con él y por qué no marcha tal y cual [...].

(12) H1: espera calla calla

H2: está lloviendo

(...)

H3: puees eso quee- no te creas tú que- y bueno yo- yo no sé ninguno ¿no? o sea a esee te lo digo porque lo sé pero supongo quee también se habrán dao casos de lo CONTRARIO

(13) H1: Yo no sabía por dónde salir y sabía que me estaban [mirando]

H2: [pues] ¿qué te iba a decir? ah sí...

4 En todos los ejemplos que constituyen reproducciones de chats se conserva la ortografía original. 
El segundo marcador discursivo analizado, es decir, bueno también dispone de tres valores pragmáticos: funciona en su modalidad deóntica, constituye un enfocador de alteridad y actúa como un marcador metadiscursivo.

Dentro de la modalidad deóntica, el citado marcador indica el acuerdo (Martín Zorraquino, Portolés 1999: 4162), pero paradójicamente con un tonema suspendido y, frecuentemente, con una reduplicación, expresa el desacuerdo (Martín Zorraquino 2008: 49). Este fenómeno se puede apreciar en los ejemplos tomados de Martín Zorraquino (2008: 48-49):

(14) H1: Mañana podríamos ir a pescar truchas.

H2: Bueno.

(15) H1: Mira, yerno, mi hija es un ángel, yo soy un encanto y tú eres un petardo.

H2: Bueno, bueno, señora...

En su segundo valor pragmático, bueno constituye un enlace entre pareceres disconformes teniendo una naturaleza transitiva entre la deóntica y la metadiscursiva (Portolés y Martín Zorraquino 1999 : 4176). Mitiga el desacuerdo y la divergencia de opiniones y, según lo denominan Briz e Hidalgo (2008: 129), se convierte en un preludio concesivo introduciendo así, de acuerdo con Calvi y Mapelli (2004), una réplica sin que esta sea interpretada como categórica o la única posible (ej. 16 recogido por Briz e Hidalgo 2008: 129) ni tampoco controvertida (ej. 17 tomado de Briz 1995: 108).

(16) H1: ¿qué te pareció la peli? ¿es genial verdad?

H2: bueno/ no sé qué decirte/ a mí me pareció un poco floja

(17) Juan no vendrá / bueno / yo no es que lo sepa seguro

Conviene añadir que las intervenciones que empiezan por bueno conllevan un tono de indecisión y duda (Llorente Arcocha 1996: 135 citada por Boyero Rodríguez 2004: 244):

(18) Sí, bueno y también para ganar dinero. ... sí, bueno, vamos a ver. Efectivamente, el gran traficante lo hace para ganar dinero. ${ }^{5}$

Por último, de acuerdo con Briz e Hidalgo (2008: 128-131), dicho marcador desempeña papeles metadiscursivos conforme al contexto y su colocación en el enunciado, a saber, señala la recepción del mensaje (ej. 19 recogido por Martín Zorraquino, Portolés 1999: 4163), introduce un discurso o una propuesta (ej. 20 de Boyero Rodríguez 2004: 248), cierra el discurso, forma una pausa (ej. 21 recopilado por Briz e Hidalgo 2008: 130), sirve para interrumpir y tomar la palabra (ej. 22 tomado de López Quero 2007: 107) o llama la atención.

(19) H1: Juan no sabe nada de esto...

H2: Bueno.

(20) H1: Bueno, ite tomas otra copa?

H2: De acuerdo...

5 Ejemplo tomado de CREA, del programa Si yo fuera presidente, 18/10/83, TVE 2. 
(21) H1: te gusta máas el americano/ hombre está claro

$\mathrm{H} 2$ : bueno no yo no tengo ninguna preferencia por nacionalidades mi preferencia es por calidades

(22) H1: bueno

$\mathrm{H} 2$ : quien se viene al agua

H1: bueno y kien me a puesto el bannnnnnnnnnnnnnnnnnnn

Cuando se ubica en el interior de un enunciado, rectifica o introduce la autocorrección (Martín Zorraquino, Portolés 1999: 4165-4166, ej. 23 ibídem), permite matizar o aclarar lo dicho (ej. 24), sirve para cambiar de tema o volver al tema después de una digresión (ej. 25) y finalmente acumula la información y ayuda en la progresión del discurso (Briz, Hidalgo 2008: 128-129).

(23) (...) Nadie puede elegir un guion, ni yo ni nadie. Bueno, quizá Jane Fonda, y tampoco lo creo

(24) (...) son unos-unos cromitos que vendían en las mermeladas de fresa / / bueno / de fresa / de mora y de todo/ (...)

(25) A: y tú ¿qué? ¿cómo estás?

B: bien / bueno voy a mirar el horno que tengo la comida a ver si después de que os invite se me quema

Bueno puede ampliar su valor pragmático a un tono enfático, aun exclamativo ${ }^{6}$, que implica enfado o impaciencia (Briz, Hidalgo 2008: 130-131) como en el ejemplo recopilado por Calvi y Mapelli (2004):

(26) ¡Bueno, a dormir!

COMBINACIÓN DE BUENO Y PUES

Por otro lado, bueno y pues pueden aparecer juntos en una intervención, más frecuentemente en este orden particular y separados por una coma: bueno, pues (27). Ahora bien, en algunas ocasiones pues aparece primero y le sigue bueno que, en cambio, no debe ser precedido por ningún signo de puntuación (28 y 29).

(27) Entonces yo he quedado en, mañana, llevar las dos llaves... para abrir. Y, bueno, pues yo creo que nada más, porque allí no había documentos de interés. ${ }^{7}$

(28) H1: ¿Quiénes dice usté, los gilimotos o los munícipes?

H2: ¿Eh? Ah, pues, bueno, a lo mejor los unos y los otros. ${ }^{8}$

(29) Y con el dicho es lo mismo. Yo lo digo y si cae mal, pues bueno, luego te puedes dis$\operatorname{culpar}^{9}(\ldots)$.

\footnotetext{
${ }^{6}$ Asemejándose al uso de la interjección bueno.

Ejemplo tomado de CREA, de la revista Tiempo, 28/05/1990: "Sólo han pillado papeles chungos".

Ejemplo tomado de CREA, del diario La Razón, 09/04/2003: Naturaleza muerta.

9 Ejemplo tomado de CREA, del programa Hablando se entiende la gente, Madrid, 01/02/91, Tele 5.
} 
A continuación, con el propósito de detectar el papel pragmático de los marcadores, se analizarán las secuencias comunicativas del corpus que provienen de las novelas de Juan Marsé Rabos de lagartija $(2002=\mathrm{RL})$ y Un día volveré $(2013=\mathrm{DV})$, Eduardo Mendoza Sin noticias de Gurb $(2010=$ NG) y La ciudad de los prodigios $(2001=\mathrm{CP})$, Carmen Posadas Pequeñas infamias $(2002=\mathrm{PI})$ y de la novela gráfica de Alfonso Zapico La ruta Joyce (2011, = RJ) y sus respectivas traducciones: Ogony jaszczurki (2003) en la traducción de Elżbieta Komarnicka, Wróce pewnego dnia (1989) de Anna Trznadel-Szczepanek, Brak wiadomości od Gurba (2010) de Magdalena Tadel, Miasto cudów (2010) de Anna Sawicka, Mate niegodziwości (2003) de Ewa Zaleska, Śladami Joyce'a (2012) de Jakub Jankowski. Tal selección se debe al carácter oral, coloquial y dialogado de los textos.

Se cotejarán los fragmentos con sus respectivas traducciones polacas para averiguar si las funciones previamente expuestas se conservan o no. Sin embargo, dadas las limitaciones espaciales del presente artículo, no se pretende llevar a cabo un exhaustivo análisis comparativo ${ }^{10}$, sino más bien observar ciertas tendencias y decisiones traductológicas.

Antes de proseguir con el estudio de las secuencias comunicativas, conviene advertir que los valores pragmáticos que desempeña cada marcador se superponen y entrecruzan, por lo tanto, los pasajes están organizados en función del valor que puede considerarse dominante.

En la mayoría de los fragmentos (diecisiete de diecinueve), bueno aparece como primero. En uno de estos pasajes, dicho marcador se utiliza en su modalidad deóntica (ej. 30), en dos se usa en calidad del enfocador de alteridad (ej. 31 y 32 ) y en los ocho restantes (ej. 33-46) desempeña el papel metadiscursivo, actúa como una pausa enunciativa, introduce un discurso, sirve para tomar palabra, hace fluir el monólogo, posibilita el cambio de tema, permite volver al tema interrumpido o cierra el discurso.

Como marcador de la modalidad deóntica, bueno expresa el acuerdo con el interlocutor, reforzado, además, por el uso de un pues metadiscursivo. En la traducción, primero aparece la partícula no que hace la aserción más enérgica ${ }^{11}$ y luego tak que manifiesta la afirmación. Por lo tanto, se transmiten ambos valores pragmáticos.

(30) - ¿Cómo dices? ¿Un qué?

- Un dragón.

- Será un lagarto. Una salamanquesa.

- Bueno, pues eso. (DV, 104)
(30) - Co takiego? Co to jest?

- Smok.

- Może gekkon?

- No tak.

Tal y como se puede apreciar a continuación, bueno suaviza una respuesta sorprendente, ya que en el fragmento (31) en vez de dar consejo el interlocutor muestra desin-

10 Las aportaciones más significativas para el estudio de partículas discursivas en polaco pueden encontrarse en las siguientes monografías: Awdiejew (2004), que examina los llamados operadores interactivos (operatory interakcyjne) en el marco de la teoría de actos de habla, y Grochowski (1997), que incluye un diccionario de locuciones funcionales (wyrażenia funkcyjne) seleccionadas por el autor.

11 Esta partícula añade un matiz expresivo particular (Stownik języka polskiego PWN). 
terés ${ }^{12}$. De modo similar, el otro marcador, empleado con su valor comentador, sirve para tomar una postura cooperativa al introducir una reacción inesperada. En cambio, en la versión polaca, bueno se traduce con un equivalente, pero en su modalidad deóntica, puesto que $w$ porzadku significa el acuerdo. En cuanto a pues, este se omite, ya que powiem $\mathrm{ci}$ solo se puede considerar la traducción de oye mediante una modulación. En la secuencia comunicativa (32), bueno mitiga la disparidad de opiniones, cuando el hablante cita la idea del interlocutor que no le conviene, y es seguido por un pues comentador. La traductora escoge la partícula cóż que introduce un comentario y atenúa el desacuerdo.

(31) - (...) ¿Qué puedo hacer, David?

- Bueno, pues oye, que te zurzan. Déjame ver la peli. (RL, 261)

(32) (...) un poco de razón ya tienes, pero ¿qué alternativa le ves? Bueno, pues quedarnos en éste. ¿Y hacer qué? $(\mathrm{NG}, 92)$
(31) - (...) Co mogę zrobić, powiedz?

- W porządku, powiem ci, niech cię thuką.

Daj mi oglądać film.

(32) (...) masz trochę racji, ale widzisz jakąś inną możliwość? Cóż, zostać tutaj. I co robić?

En cambio, en los siguientes fragmentos bueno desempeña un papel metadiscursivo; en el ejemplo (33) este introduce el discurso en un corto capítulo Los nazis y Finnegans Wake marcando el inicio de la historia, mientras que pues constituye un conector consecutivo que indica la continuidad con la sección anterior en la cual el narrador menciona que hablará de la época de la ocupación nazi. Igualmente, en el fragmento (34), el primer marcador inicia una corta historia y el segundo la une con el tema anterior. Los traductores polacos optan por un equivalente acuñado no więc que, al mismo tiempo, inicia una intervención y contribuye a la cohesión del discurso, ya que alude al contexto precedente (Słownik języka polskiego PWN).

(33) Bueno, pues el caso es que un oficial alemán pasó frente a la librería de Sylvia Beach, y se encaprichó con un libro en particular. (RJ, 128)

(34) - Ya sabes que mi mujer está enferma y que la he mandado unos días del pueblo... - Sí.

- Bueno, pues la otra noche me di una vuelta por el barrio chino. Uno tiene derecho a distraerse un poco, sin que la parienta se entere. (DV, 95)
(33) No więc, było to tak, że pewien niemiecki oficer przechodził obok księgarni Sylvii Beach i szczególnie zainteresowała go jedna książka.

(34) - Wiesz, że moja żona jest chora i że wysłałem ją na parę dni na wieś...

- Tak...

- No więc któregoś wieczoru przeszedłem się do „chińskiej” dzielnicy. Człowiek ma prawo się rozerwać, byle baba nie wiedziała (...).

En la siguiente secuencia, bueno actúa como una pausa enunciativa proporcionando el tiempo para concentrarse y encontrar una manera de expresar los pensamientos, mientras que pues, de nuevo, aparece como conector consecutivo que enlaza la parte anterior con lo que le sigue. De manera similar a los fragmentos anteriores, en la traducción polaca se usa el mismo equivalente, ya que no suele emplearse cuando el hablante no sabe

12 La expresión que te zurzan debe traducirse como spadaj o idź do diabła y no como niech cię ttukq (= que te peguen). 
muy bien qué decir, mientras que więc mantiene la continuidad contextual (Stownik języka polskiego $P W N$ ).

(35) Y qué más, qué más... Bueno, pues que esta señora vive realquilada. $(\mathrm{RL}, 29)$
(35) I co jeszcze, co jeszcze... No więc ta pani podnajmuje domek.

En dos fragmentos (36 y 37), bueno sirve para tomar la palabra. En el primer diálogo, le acompaña un pues comentador que introduce una reacción no esperada por el interlocutor y en la versión polaca se traduce con no que suele aparecer al inicio de una respuesta (Słownik języka polskiego PWN), no necesariamente para "apoyar el acto cortés". En cambio, en la conversación 37 bueno adquiere, además, un valor intensificador que resalta la impaciencia de la madre que ya no quiere seguir hablando, mientras que pues une el valor continuativo con el valor metadiscursivo, en concreto exhortativo. La traductora elige $w$ takim razie que tiene el papel de un conector consecutivo y, por lo tanto, constituye un equivalente acuñado de pues solo en esta función. De ahí que resulte extraño su uso en el contexto.

(36) - Has vuelto a caerte de esa dichosa bicicleta, a que sí. No me mientas.

David piensa la respuesta un rato.

- Bueno, pues sí. (RL, 106)

(37) - Ya estaba muy pirada, la pobre abuela.

- Bueno, pues que te lo cuente tu padre cuando vuelva. $[\ldots]$ Y basta de preguntas. $(\mathrm{RL}, 134)$
(36) - Znowu spadłaś z tego przeklętego roweru, może nie? Tylko mi nie kłam.

David myśli przez chwilę nad odpowiedzią.

- No tak, spadłam.

(37) - Już była całkiem niepoczytalna, biedna babcia.

- W takim razie niech ci to ojciec opowie, jak wróci (....... I dość tych pytań.

En los fragmentos (38), (39) y (40), bueno, en su función metadiscursiva, permite la progresión temática. Entre tanto, pues desempeña el papel de un conector consecutivo en el primero y el tercero, a diferencia del segundo en el que sirve de comentador. Otóż resulta un buen equivalente, ya que introduce un comentario relacionado con el tema anterior, aunque no es una palabra frecuentemente utilizada en el habla. En cambio, no tak parece más bien responder a la pregunta retórica prawda? Que contribuir a la progresión temática o indicar la consecuencia. No obstante, la partícula więc, equivalente de entonces, se emplea como conector consecutivo. En cuanto al último fragmento, la expresión no, dobrze no resulta del todo acertada, dado que el adverbio polaco muestra el acuerdo, un valor pragmático ausente en el original. 
(38) Usted sufre una enfermedad incurable, eso (se lo habrán diagnosticado; cáncer, ¿verdad? Bueno, pues entonces le alegrará saber que no morirá de... (PI, 93)

(39) Como tú recordarás, acudimos a ella para conseguir un filtro que le permitiera encontrar una réplica de su mujer ideal. Bueno, pues para mi gran alivio, ha sucedido algo sorprendente (...). (PI, 182)

(40) De pronto un ayudante de campo se apea del caballo y entra en la peluquería; aún me parece estar oyendo el taconeo de las botas y el ruido que hacían las espuelas en las baldosas. Bueno, pues me mira y me dice: ¿Está el dueño? (CP, 58)
(38) Cierpi pan na nieuleczalną chorobę, pewnie rozpoznali u pana raka, prawda? No tak, więc ucieszy pana pewnie wiadomość, że nie umrze pan z powodu...

(39) Pewnie pamiętasz, że poszliśmy do niej po miłosny napój, dzięki któremu Carlos miał spotkać sobowtóra swego ideału kobiety. Otóż ku mojej wielkiej uldze sprawy przybrały zaskakujący obrót (...).

(40) Nagle ordynans zsiadł z konia i wchodzi do zakładu; do tej pory pamiętam stukot butów do konnej jazdy na bruku i pobrzękiwanie ostróg na posadzce. No, dobrze, patrzy na mnie i mówi: Jest szef?

En la siguiente secuencia comunicativa tanto bueno como pues en su carácter metadiscursivo permiten volver al tema interrumpido, en (41) a la historia de un piloto inglés, después de una digresión sobre la lengua inglesa y en (42) a la historia de una perla tras la digresión sobre el aspecto físico de su propietario. En la versión polaca se opta de nuevo por no więc (dividido, o no, por coma) gracias al cual se reanuda al tema o la situación precedente.

(41) Algo aprendió, algo aprendió... ¿Por dónde íbamos? Ah, sí. Bueno, pues yo hice un trabajo irreprochable con ese piloto (...). (RL, 186)

(42) ¿Han visto a este caballero tan respetable (...)? Bueno, pues ese caballero me ha regalado una perla (...). (PI, 95)
(41) Trochę się nauczyła, trochę się nauczyła... Na czym to skończyliśmy? Ach, tak. No więc ja w stosunku do tego pilota wykonałem swoje zadanie bez zarzutu (...).

(42) Widzieli panowie tego szacownego starszego pana (...)? (...) No, więc ten dżentelmen podarował mi prawdziwą perłę (...).

En su calidad metadiscursiva, bueno puede también iniciar un cambio temático como se puede apreciar en el fragmento 43. El otro marcador se emplea con el objetivo de abrir un nuevo comentario. El adverbio nieważne, empleado por la traductora, efectivamente, permite pasar a otro tema, pero descartando como poco importante la información precedente, lo cual no acontece en el original.

(43) (...) una que hay sobre la mesa, una foto divina, y reciente además, ¿no? Bueno, pues me he quedado asombrada, hay que ver qué increíble es la genética, tesoro (...). (PI, 205)
(43) (...) o tym na stole, piękne zdjęcie, poza tym całkiem aktualne, prawda? Nieważne, kompletnie osłupiałam, jednak geny to coś nieprawdopodobnego, skarbie (...).

Finalmente, bueno sirve para cerrar el discurso y le sigue un pues consecutivo que enlaza lo anteriormente dicho con cierto tipo de conclusiones. Los traductores polacos escogen dos versiones (no y no więc) que, sin embargo, tienen la misma función: mantienen la continuidad temática conforme a lo que se ha comentado anteriormente. 
(44) Parece que él y sus amigos lo habían seguido (...) seguramente para reírse de él, dicen que iba como sonámbulo y llorando, pobre hombre. Bueno, pues a mi hijo le causó una impresión tremenda. (RL, 167)

(45) Bueno, pues ya está. Joyce murió (...). (RJ, 192)

(46) Bueno, pues ya está. Esto es el final. No sé qué más decir. (RJ, 201)
(44) Zdaje się, że on i jego koledzy (...) chodzili za nim (...) na pewno żeby się z niego wyśmiewać. Podobno szedł jak nieprzytomny i płakał, biedny człowiek. No więc, na moim synu to wywarło potworne wrażenie.

(45) No, to by było na tyle. Joyce umarł (...).

(46) No, to już. To koniec. Nie wiem, co dodać.

Dos de todos los fragmentos recogidos incluyen los dos marcadores en el orden invertido y en ambos el primero, es decir, pues desempeña el papel de una pausa. Respecto a bueno, este se utiliza en calidad de metadiscursivo en el diálogo (47) para cambiar de tema, mientras que en la situación (48) se lo emplea para introducir un discurso. En las dos traducciones aparece no que, como se ha mencionado con anterioridad, abre una intervención cuando el hablante está vacilante. Dicho adverbio está seguido por una palabra que expresa el acuerdo, respectivamente, dobra y tak. En ambos casos, resultaría más adecuado optar por no cóż que puede, por un lado, dar a entender que el interlocutor está cerrando un tema sin añadir cualquier comentario (ej. 47) y, por otro, mostrar que se habla a regañadientes (ej. 48).

(47) - Se necesita ser merluzo para decir estas cosas...

- Pues bueno - observa el bulto del puño de David metido en el bolsillo del pantalón y añade-: Oye, ¿qué vas a hacer con el mechero del inspector? (RL, 298)

(48) La charla fue algo así como esto...

- Pues... Bueno, el libro no está en inglés, (...). (RJ, 195)
(47) - Chyba trzeba być świrniętym, żeby coś takiego wymyślić...

- No, dobra. - Patrzy na pięść Davida wybrzuszającą kieszeń spodni i dodaje: - No i co w końcu zrobisz z zapalniczką inspektora?

(48) Gadka była mniej więcej taka...

- No... Tak, książka oczywiście nie jest po angielsku (...).

CONCLUSIONES

Tal y como apunta Portolés (1998: 151), a la hora de traducir los marcadores discursivos los traductores deben centrarse particularmente en las instrucciones de procesamiento proporcionadas por estos, dado que, en realidad, no existen buenas soluciones prefabricadas. No obstante, en los fragmentos analizados, algunos equivalentes prevalecen, no więc (usado seis veces) y no (utilizado tres veces). Si bien en los originales los dos marcadores españoles pueden desempeñar diversas funciones, incluso a la vez, el abanico de funciones de los equivalentes polacos en muchas ocasiones se corresponde con el original. En cuanto a las dos versiones más frecuentes, la partícula no empieza un enunciado, especialmente cuando el hablante está buscando las palabras adecuadas, precede a las órdenes, pero también marca la continuidad, así como więc. Este último 
contribuye, además, a la cohesión del discurso aludiendo al contexto precedente. En algunos casos (secuencias 31, 37, 43, 47, 48), la traducción no transmite el mismo valor que el original, en el primer, cuarto y quinto caso, puesto que se sitúa en la modalidad deóntica en vez de metadiscursiva, mientras que en el segundo se limita a expresar la consecuencia sin destacar el mandato y en el tercero añadiendo un juicio ausente del original. A pesar de estas cinco modificaciones, en los demás fragmentos (catorce de los diecinueve), el papel de bueno y pues se ha descodificado correctamente y se ha expresado, si bien parcialmente, en la versión polaca.

BIBLIOGRAFÍA:

\section{Fuentes primarias}

MARSÉ Juan, 1989, Wrócę pewnego dnia, Warszawa: Państwowy Instytut Wydawniczy.

MARsÉ Juan, 2002, Rabos de lagartija, Barcelona: Planeta DeAgostini.

MARSÉ Juan, 2003, Ogony jaszczurki, Warszawa: Świat Książki.

MARsé Juan, 2013, Un día volveré, Barcelona: Debolsillo.

Mendoza Eduardo, 2001, La ciudad de los prodigios, Madrid: Bibliotex S.L.

Mendoza Eduardo, 2010a, Brak wiadomości od Gurba, Kraków: Znak.

Mendoza Eduardo, 2010b, Miasto cudów, Kraków: Znak.

Mendoza Eduardo, 2010c, Sin noticias de Gurb, Pamplona, Leer-e.

Posadas Carmen, 2002, Pequeñas infamias, Barcelona, Editorial Planeta.

Posadas Carmen, 2003, Małe niegodziwości, Warszawa: Warszawskie Wydawnictwo Literackie Muza.

ZAPICO Alfonso, 2011, La ruta Joyce, Bilbao: Astiberri.

ZAPICO Alfonso, 2012, Śladami Joyce'a, Warszawa: Timof Comics.

\section{Fuentes secundarias}

AwDIEJEw Aleksy, 2004, Gramatyka interakcji werbalnej, Kraków: Wydawnictwo Uniwersytetu Jagiellońskiego.

BOyero RodríGuez María José, 2004, Aportación al estudio de los marcadores conversacionales que intervienen en el desarrollo del diálogo, Madrid: Universidad Complutense de Madrid, http:// biblioteca.ucm.es/tesis/fll/ucm-t25099.pdf (fecha de consulta: junio 2017).

BRIz Antonio, 1995, La atenuación en la conversación coloquial. Una categoría pragmática, (in:) El español coloquial, Actas del I Simposio sobre análisis del discurso oral, Luis Cortés Rodríguez (coord.), Almería: Servicio de Publicaciones de la Universidad de Almería, 103-122.

Briz Antonio, Hidalgo Antonio, 2008, Conectores pragmáticos y la estructura de la conversación, (in:) Los marcadores del discurso. Teoría y análisis, María Antonia Martín Zorraquino, Estrella Montolío Durán (red.), Madrid: Arco/Libros, S.L., 121-142.

Calvi Maria Vittoria, MaPelli Giovanna, 2004, Los marcadores bueno, pues, en fin, en los diccionarios de español e italiano, Artifara 4, (gennaio-giugno 2004), sezione Monographica, http://www.artifara.com/rivista4/testi/marcadores.asp, sin paginar (fecha de consulta: junio 2017).

Grochowski Maciej, 1997, Wyrażenia funkcyjne. Studium leksykograficzne, Kraków: Wydawnictwo Instytutu Języka Polskiego PAN.

LÓPEZ QUERo Salvador, 2007, Marcadores discursivos y cortesía en la conversación virtual en español, Language Design 9, 93-112.

Martín Zorraquino María Antonia, 2008, Los marcadores del discurso desde el punto de vista gramatical, (in:) Los marcadores del discurso. Teoría y análisis, María Antonia Martín Zorraquino, Estrella Montolío Durán (red.), Madrid: Arco/Libros, S.L., 19-53. 
Martín Zorraquino María Antonia, Portolés José, 1999, Los marcadores del discurso, (in:) Gramática descriptiva de la lengua española, Ignacio Bosque, Violeta Demonte (dirs.), Madrid: Espasa Calpe, 4051-4214.

Montolío Durán Estrella, 2008, Conectores de la lengua escrita: contraargumentativos, consecutivos, aditivos y organizadores de la información, Barcelona: Editorial Ariel.

Otaola Olano Concepción, 2004, Lexicología y semántica léxica. Teoría y aplicación a la Lengua Española, Madrid: Ediciones Académicas, S.A.

PORTOLÉS José, 1998, Marcadores del discurso, Barcelona: Ariel.

WitTGenstein Ludwig, [1953] 1999, Investigaciones filosóficas, Barcelona: Altaya.

\section{Diccionarios y bancos de datos}

Real Academia Española: Banco de datos (CREA), http://www.rae.es (fecha de consulta: marzo 2020). SAntos Río Luis, 2003, Diccionario de partículas, Salamanca: Luso-Española de Ediciones.

Słownik języka polskiego PWN, https://sjp.pwn.pl/ (fecha de consulta: junio 2017). 